\title{
The Exponential Distribution Applied to Nonequidistantly Sampled Cardiovascular Time Series
}

\author{
H. G. Van Steenis and J. H. M. Tulen \\ Department of Psychiatry, Erasmus University Rotterdam, The Netherlands
}

Received August 11, 1995

\begin{abstract}
Beat-to-beat fluctuations in cardiovascular time series comprise different frequency components which can be employed to describe autonomic regulatory processes. The Exponential Distribution (ED) is presented here as a specific time-frequency distribution which has the potential to describe the time-related changes in the frequency content of these cardiovascular fluctuations. The ED has as advantage that it gives a good suppression of the cross terms, a characteristic feature of bilinear time-frequency distributions. An implementation to apply the ED to nonequidistantly sampled cardiovascular time series is provided. Applications of the ED to various clinical and experimental human cardiovascular time series show that the ED can be an important aid to describe and interpret time-varying frequency components of cardiovascular signals such as heart rate, interbeat interval, blood pressure, and respiration. ๑1 1996 Academic Press, Inc.
\end{abstract}

\section{INTRODUCTION}

The conventional method to study the dynamics of cardiovascular control mechanisms is to estimate the variability spectra of the beat-to-beat fluctuations in heart rate (HR) and blood pressure (BP). These fluctuations in HR and BP are usually characterized by three spectral peaks in a frequency range of 0.01 to $0.50 \mathrm{~Hz}$ : a low frequency peak around $0.04 \mathrm{~Hz}$, a mid frequency peak around $0.1 \mathrm{~Hz}$, and a high frequency peak between $0.20-0.35 \mathrm{~Hz}$. These peaks are believed to contain differential information related to either sympathetic and/or parasympathetic processes within the cardiovascular control system (1-4), whereas respiratory-related high frequency fluctuations in HR particularly reflect cardiac vagal (parasympathetic) tone, low, and mid frequency fluctuations in HR and, especially, BP may reflect sympathetic processes.

To estimate HR spectra and spectra of other cardiovascular signals, several methods have been developed. Most of these methods are based on equidistant sampling of the cardiovascular signals, in order to produce various time series of cardiovascular quantities such as interbeat intervals or systolic BP (SBP). Spectra can then be calculated using a fast Fourier transform. However, HR is 
a frequency modulated signal (5), whereas SBP and diastolic BP (DBP) are frequency and amplitude modulated signals (6). We recently presented a method to compute spectra of cardiovascular time series by means of nonequidistant sampling of the R-waves (7) based on the assumptions of Rompelman (5) and Mulder (6). Nonequidistant sampling of the HR signal or other cardiovascular signals guarantees that whenever time relationships between signals have to be studied (such as between HR and SBP), there are no relative time shifts between the time series or the relative time shifts are constant over time. Computation of variability spectra and cross-spectra by means of nonequidistant sampling of the cardiovascular signals have the advantage that these methods take into account both the frequency and amplitude modulation properties of these signals.

Spectral analysis is usually performed on consecutive time-segments ranging from 2 to $5 \mathrm{~min}$, under the assumption that these time-segments can be considered stationary. But even on this time-scale, the spontaneous cardiovascular fluctuations are, in general, not completely stationary. This makes the spectra of the consecutive segments less appropriate to examine changes in frequency components over time. For each segment, the time-varying frequency components will accumulate into wide frequency bands within the overall power spectrum. Furthermore, the high segment-to-segment variations in the power of the different frequency components over time (8) suggest that a more detailed analysis of time-frequency relationships is warranted. These relationships can be described with a (joint) time-frequency distribution (TFD). A TFD describes the frequency changes of the signal as a function of time, while allowing nonstationarities. At each instant of time, a TFD shows the frequencies that exist at that time. A well-known TFD is the Wigner-Ville Distribution (WVD; 9-14). Novak and Novak (15) were the first to apply the WVD to cardiovascular time series. In this paper, we will explore the possibilities of the exponential distribution $(\mathrm{ED} ; 16)$ to describe time-frequency relationships of various cardiovascular time series. The ED is a modified WVD, which diminishes the effects of the so-called cross terms. These cross terms are artefacts in a TFD due to the bilinearity of the TFD; cross terms are considered to be a drawback of the WVD.

Our aim of this paper is to apply the exponential distribution to nonequidistantly sampled cardiovascular time series. The implementation of the method into a computer program is provided. Furthermore, the applicability of the method to study details of cardiovascular time-frequency relationships is illustrated on both clinical and experimental human data.

\section{THEORY}

\subsection{Spectral Analysis of Nonequidistant Cardiovascular Time-Series}

The estimation of HR variability (HRV) spectra based on nonequidistant sampling of the R-wave incidences was first introduced by Rompelman (5). The R-waves are detected and represented by Dirac delta pulses $\delta\left(t-t_{i}\right)$ at the incidence times $t_{i}, i=1,2, \ldots, N$. These delta pulses form the signal: 


$$
p(t)=\sum_{i=1}^{N} \delta\left(t-t_{i}\right)
$$

Such a series of consecutive R-wave incidences is called a HR signal or HR time series.

The Fourier transform of the function $p(t)$ is:

$$
X_{c}\left(f_{k}\right)=\int_{-\infty}^{\infty} p(t) \cdot e^{-2 \pi j f_{k} t} d t=\sum_{i=1}^{N} e^{-2 \pi j f_{k} t_{i}}
$$

in which $f_{k}=k / T, k=0,1, \ldots$, and $T$ is the total record time. The spectrum $P_{C}$ of the function $p(t)$ is (17):

$$
P_{C}\left(f_{k}\right)=\frac{2}{T} \cdot X_{C}\left(f_{k}\right) \cdot X_{C}^{*}\left(f_{k}\right)
$$

and is estimated using the right part of Eq. [2]. This spectrum is based on nonequidistant sampling: the delta pulses are occurring at the R-wave incidences. The spectrum $P_{C}$ is called the spectrum of counts (SOC).

Let $p(t)$ be a HR time series of consecutive R-wave incidences at times $t_{i}$, $i=1,2, \ldots, N$. The time-intervals $\Delta t_{i}$ between the R-waves or interbeat intervals $I_{i}$ (IBIs) are defined by $I_{i}=\Delta t_{i}=t_{i}-t_{i-1}$ for $i=1,2, \ldots, N$ (the first interval starts at $\mathrm{R}$-wave incidence $\left.t_{0}\right)$. The total record time $T$ is $\Sigma\left\{\Delta t_{i}, i=\right.$ $1,2, \ldots, N\}$. The mean sample interval $\Delta t$ or mean IBI $I$ is $T / N$. The "sample frequency" is defined by $f_{S}=1 / I=N / T$ (this is also called the "mean HR") The "Nyquist frequency" is defined by $f_{N}=f_{S} / 2$. The frequency resolution is $\Delta f=1 / T$.

Prior to the calculation of the spectrum of a time series, it is customary to subtract the DC of the time series. In case of a HR signal, DC-correction is performed by subtracting a sequence of $N$ delta functions from the original delta series $p(t)$ in Eq. [1]. These delta functions are placed at equal intervals $I$, the mean IBI. The function $p(t)$ then becomes (6):

$$
p(t)=\sum_{i=1}^{N} \delta\left(t-t_{i}\right)-\sum_{i=1}^{N} \delta(t-i \cdot I) .
$$

At the R-wave incidences, samples of other cardiovascular signals can be obtained: the SBP or DBP can be determined, the IBI since the last R-wave incidence can be calculated, or a sample of the respiration (RSP) signal can be taken. In this way, a collection of data points on a beat-to-beat basis is obtained. Such a series is called a cardiovascular time series. Mulder (6) introduced a method to calculate the spectrum of these nonequidistantly sampled signals (see also van Steenis et al. (7)).

Let $\left\{x_{i}, i=1,2, \ldots, N\right\}$ be the samples of a cardiovascular signal taken at the R-wave incidences $t_{i}, i=1,2, \ldots, N$. The R-wave incidences are again represented by the delta functions $\delta\left(t-t_{i}\right)$, but now they have weights $x_{i} \cdot \Delta t_{i}$ in which $\Delta t_{i}$ is the time interval $t_{i}-t_{i-1}$. Together they form the function $p(t)$ : 


$$
p(t)=\sum_{i=1}^{N} x_{i} \cdot \Delta t_{i} \cdot \delta\left(t-t_{i}\right)
$$

The Fourier transform of the function $p(t)$ is:

$$
X\left(f_{k}\right)=\int_{-\infty}^{\infty} p(t) \cdot e^{-2 \pi j f_{k} t} d t=\sum_{i=1}^{N} x_{i} \cdot \Delta t_{i} \cdot e^{-2 \pi j f_{k} t_{i}} .
$$

The spectrum $P$ of the function $p(t)$ is:

$$
P\left(f_{k}\right)=\frac{2}{T} \cdot X\left(f_{k}\right) \cdot X^{*}\left(f_{k}\right)
$$

and is estimated using the right part of Eq. [6].

DC- correction is applied by subtracting a weighted mean of the samples $x_{i}$ from each sample. This weighted mean has the form:

$$
x_{W}=\frac{1}{T} \cdot \sum_{i=1}^{N} x_{i} \cdot \Delta t_{i}
$$

and Eq. [6] becomes:

$$
X\left(f_{k}\right)=\sum_{i=1}^{N}\left(x_{i}-x_{W}\right) \cdot \Delta t_{i} \cdot e^{-2 \pi j f_{k} t_{i}}
$$

\subsection{The Wigner-Ville Distribution}

The traditional method to estimate the power spectrum of a stationary stochastic process $x(t)$ is the calculation of the Fourier transform of the autocorrelation function (ACF) of $x(t)$ :

$$
S_{x}(f)=\int_{-\infty}^{\infty} R_{x}(\tau) \cdot e^{-2 \pi j f \tau} d \tau,
$$

in which $R_{x}(\tau)=E\left[x^{*}(t) \cdot x(t+\tau)\right]$ is the ACF of the process $x(t)(*$ denotes the complex conjugate) (17). For a stationary process $x(t)$, the ACF $R_{x}(\tau)$ is only dependent on the time lag $\tau$ and is not dependent on time $t$. This property does not hold for nonstationary stochastic processes. In that case, the ACF of a stochastic process $x(t)$ becomes $R_{x}(t, \tau)=E\left[x(t+\tau / 2) \cdot x^{*}(t-\tau / 2)\right]$ (17). Substituting this ACF in Eq. [10] yields a spectral representation of $x(t)$, given by:

$$
S_{x}(t, f)=\int_{-\infty}^{\infty} R_{x}(t, \tau) \cdot e^{-2 \pi j f_{\tau}} d \tau .
$$

It follows that:

$$
S_{x}(t, f)=E\left[\int_{-\infty}^{\infty} x\left(t+\frac{\tau}{2}\right) \cdot x *\left(t-\frac{\tau}{2}\right) \cdot e^{-2 \pi j f \tau} d \tau\right]=E\left[W_{x}(t, f)\right],
$$

in which: 


$$
W_{x}(t, f)=\int_{-\infty}^{\infty} x\left(t+\frac{\tau}{2}\right) \cdot x *\left(t-\frac{\tau}{2}\right) \cdot e^{-2 \pi j f \tau} d \tau
$$

$(13,17)$. The function $W_{x}(t, f)$ is called the Wigner distribution (WD) of the stochastic process $x(t)(9,11,13)$.

The discrete time equivalent of Eq. [13] for a random process $x(t)$ is (18):

$$
W_{x}(n \Delta t, f)=2 \Delta t \cdot \sum_{i=-\infty}^{\infty} x(n \Delta t+i \Delta t) \cdot x^{*}(n \Delta t-i \Delta t) \cdot e^{-4 \pi j \cdot i \Delta t \cdot f}
$$

$(n=\ldots,-2,-1,0,1,2, \ldots)$, in which $\Delta t$ is the sample interval. $f_{S}=1 / \Delta t$ is the sample frequency. This function is periodic, with period $f_{1}=1 / 2 \cdot d t=$ $f_{S} / 2$. This means that frequency components of the signal outside the frequency range $\left[-f_{1} / 2, f_{1} / 2\right]$ will be folded within this region, i.e., aliasing will occur. There are two methods to avoid this $(12,19)$ : $(1)$ make sure that the frequency components outside $\left[-f_{1} / 2, f_{1} / 2\right]$ are zero; this can be done by oversampling the signal by, at least, a factor four of the Nyquist frequency, which is the highest frequency in the signal, i.e., $f_{1} / 2 \geq f_{N}$ and $f_{S} \geq 4 \cdot f_{N}$; or (2) make sure that the negative frequency components are zero; in this case the frequency components in the region $\left[f_{1} / 2, f_{1}\right]$ are zero and cannot disturb the frequency components in the region $\left[0, f_{1} / 2\right]$; this can be done by using analytical signals. An analytical signal is a signal without negative frequency components. It is the dual of causal signals in the time domain (17). Analytical signals can be sampled at a rate which is at least twice the Nyquist frequency, i.e., $f_{S} \geq 2 \cdot f_{N}$. In fact, the sample frequency is doubled by producing an imaginary part to the real nonanalytical signal in order to get the analytical form (19). For an analytical stochastic process $z(t)$, the function $W_{z}(t, f)$ is called the Wigner-Ville distribution (WVD; 10, 11, 13):

$$
W_{z}(t, f)=\int_{-\infty}^{\infty} z\left(t+\frac{\tau}{2}\right) \cdot z^{*}\left(t-\frac{\tau}{2}\right) \cdot e^{-2 \pi j f_{\tau}} d \tau
$$

There is a second reason to use analytical signals (19): real nonanalytical signals can produce low-frequency artefacts in the Wigner distribution. These are a result of interactions between positive and negative frequency components. Annihilation of the negative frequency components by using the analytical form of a real signal prevents these interactions. The interactions between positive and negative frequencies are comparable to the appearance of "ghost" curves between two positive components.

\subsection{Properties of TFDs}

A TFD should reflect the energy distribution of the signal, both over time and frequency. Therefore, a set of properties has been formulated which should apply to an ideal TFD $(13,14,20)$ : (P1) the TFD is real-valued; $(\mathrm{P} 2)$ integration over both time and frequency yields the total energy of the signal; (P3) integration over time yields the spectral density of the signal; (P4) integration over frequency 
yields the instantaneous power of the signal; (P5) averaging over frequency yields the instantaneous frequency; (P6) averaging over time yields the group delay; (P7) the TFD satisfies the weak finite time support: if the signal is zero outside a time segment, then the TFD is zero outside this time segment; (P8) the TFD satisfies the weak finite frequency support: if the signal is zero outside a frequency segment, then the TFD is zero outside this frequency segment; (P9) the TFD is positive. The short-time Fourier transform (STFT; 21) only satisfies P1 and P9; the WVD satisfies all except P9 $(13,14)$.

\subsection{Cross Terms and the Exponential Distribution}

A limitation of the WVD is the appearance of spectral cross terms, or "ghost" curves, in the time-frequency domain. These cross terms are artefacts, which are due to the bilinear nature of the WVD (Eq. [15]) $(13,14)$. To understand this notion we work out the WVD of the sum of two pure sine waves (14):

$$
z(t)=A_{1} \cdot e^{2 \pi j f_{1} t}+A_{2} \cdot e^{2 \pi j f_{2} t} .
$$

The WVD of this analytical function consists of two autoterms and a cross term $(\delta(\cdot)$ is the Dirac delta function):

$$
W(t, f)=A_{1}^{2} \cdot \delta\left(f-f_{1}\right)+A_{2}^{2} \cdot \delta\left(f-f_{2}\right)+\text { cross term. }
$$

The cross term has the following form:

$$
\text { cross term }=2 A_{1} A_{2} \cdot \delta\left(f-\frac{\left(f_{1}+f_{2}\right)}{2}\right) \cdot \cos \left(2 \pi\left(f_{2}-f_{1}\right) t\right) .
$$

The first two terms on the right hand side of Eq. [17] are the autoterms; they are the expected components in the time-frequency plane at the frequencies $f_{1}$ and $f_{2}$ of the sine waves (compare this with the power spectrum of a sine wave). The resulting cross term, given in Eq. [18], is located halfway between the two autoterms and the form of the cross term is sinusoidal. The cross terms in the WVD of a signal can also occur at the position of the autoterms; they obscure the true time-frequency distribution and they hamper the interpretation of the distribution.

An approach to diminish the cross terms is the use of the ED, or Choi-Williams distribution, introduced by Choi and Williams (16). In comparison to the WVD, the ED has lower cross terms amplitudes, but it contains the same information in the autoterms as the WVD (13). This is achieved by convoluting the function $z(t+\tau / 2) \cdot z^{*}(t-\tau / 2)$ in Eq. [15], as a function of $t$, with an exponential kernel which has the form:

$$
K_{\sigma}(t, \tau)=\sqrt{\frac{\sigma}{4 \pi \tau^{2}}} \cdot e^{-\sigma t^{2} / 4 \tau^{2}}
$$

in which $\sigma$ is a parameter. For an analytical stochastic process $z(t)$ the ED has the form: 


$$
E_{z}(t, f)=\int_{-\infty}^{\infty}\left\{\int_{-\infty}^{\infty} K_{\sigma}(u-t, \tau) \cdot z\left(u+\frac{\tau}{2}\right) \cdot z^{*}\left(u-\frac{\tau}{2}\right) d u\right\} \cdot e^{-2 \pi j f \tau} d \tau .
$$

For large $\sigma$, the ED is almost equal to the WVD. Smaller values of $\sigma$ will suppress the cross terms. $\sigma=2$ has been found to be a good compromise for our applications. The ED satisfies the properties P1 through P6 (section 2.3). If $\sigma$ is sufficiently large, the ED will also satisfy $\mathrm{P} 7$ and $\mathrm{P} 8(13,14,16)$.

\subsection{The Discrete Exponential Distribution}

The discrete exponential distribution (DED) of a discrete analytical time series $\{\mathrm{z}[n], n=1,2, \ldots, N\}$ is defined by $(13,16)$ :

$$
E(n, k)=2 \cdot \sum_{i=-L_{1}}^{L_{1}}\left\{\sum_{m=-M_{1}}^{M_{1}} \sqrt{\frac{\sigma}{4 \pi i^{2}}} \cdot e^{-\sigma m^{2} / 4 i^{2}} \cdot k[n+m, i]\right\} \cdot e^{-4 \pi j \cdot i k / L},
$$

in which the kernel function $k[n, i]$ is defined by:

$$
k[n, k]=v[i] \cdot z[n+i] \cdot v^{*}[-i] \cdot z^{*}[n-i],
$$

for $n=1,2, \ldots, N, k=0,1, \ldots, L-1$, and $L=2 L_{1}+1, L$ must be odd. $L$ can be equal to $N . v[i]$ is a window function of odd length $L . v[i]$ is zero outside the segment $\left[-L_{1}, L_{1}\right] . \sigma$ is a parameter. $M=2 M_{1}+1, M$ must be odd, and $M$ can be equal to $L$. The factor 4 in the exponent and the factor 2 before the summation in Eq. [21] are the result of the transformation of the continuous $\tau / 2$ into the discrete variable $i$.

\section{Methods}

\subsection{Implementation of the Exponential Distribution}

The ED can only be applied to equidistantly sampled analytical signals. HR time series and cardiovascular time series are nonequidistant time series (both series will be called cardiovascular time series from now on). In order to calculate the DED of a cardiovascular time series, this series has to be transformed into an equidistant analytical time series. Let $p(t)$ be a cardiovascular time series, according to section 2.1. The first step is to calculate the complex Fourier transform, using Eq. [2] or [9], of this $p(t)$. This results in an equidistant complex series in the frequency domain. This series can be transformed back to the time domain with the well-known inverse discrete Fourier transform (DFT). The number of $\mathrm{R}$-waves in $p(t)$ and accordingly the number of complex data in the frequency domain is generally not a power of two. Furthermore, it can be an odd number. Therefore, a fast Fourier transform (FFT) cannot be used here. An efficient method to calculate the DFT or the inverse DFT of an arbitrary number of data is the chirp Z-transform (CZT) resp. inverse chirp Z-transform (22). So, the second step is to apply the inverse CZT to the complex series in the frequency domain in order to get the desired equidistant time series in the 
time domain. It guarantees the same time resolution as the original time series. And the power spectrum of the equidistant series, calculated with the DFT, will be equal to the power spectrum of the nonequidistant series, calculated with Eq. [3] or [7]. The next step is to calculate the analytical form of the resulting equidistant cardiovascular time series. One method, using the DFT, in this case the CZT, is described by Boashash and Reilly (23). In short, this method calculates the complex Fourier transform of the time series, annihilates the negative frequency components and multiplies the positive frequency components by two, and transforms the result back to the time domain. An alternative way to produce the analytical form is to use the Hilbert transform (17). A FIR filter implementation of the Hilbert transform is discussed by Boashash and Black (18). The DED of the resulting equidistant analytical cardiovascular time series can be calculated as follows. Let $\{z[n], n=1,2, \ldots, N\}$ be a discrete equidistant analytical cardiovascular time series and $\left\{v[i], i=-L_{1}, \ldots, L_{1}\right)$ a window function of odd length $L=2 L_{1}+1 . T$ is the total measuring time and $\Delta t=T / N$ is the sample interval. $T^{\prime}$ is the duration of the window function, i.e., $T^{\prime}=L \cdot \Delta t$. Define:

$$
k_{n}[i]=\sum_{m=-M_{1}}^{M_{1}} \sqrt{\frac{\sigma}{4 \pi i^{2}}} \cdot e^{-\sigma m^{2} / 4 i^{2}} \cdot k[n+m, i]
$$

(see Eq. [22]) and

$$
C_{L}[\alpha]=e^{2 \pi j \cdot \alpha \cdot L_{1} / L} .
$$

Define $k^{\prime}=2 k$ and rewrite Eq. [21]:

$$
E^{\prime}\left(n, k^{\prime}\right)=E\left(n, k^{\prime} / 2\right)=2 \cdot C_{L}\left[k^{\prime}\right] \cdot \sum_{l=0}^{L_{1}} k_{n}\left[l-L_{1}\right] \cdot e^{-2 \pi j \cdot l k^{\prime} / L},
$$

for $k^{\prime}=0,1, \ldots, L-1$. Thus, the DED consists of the DFTs of the series $\left\{k_{n}[i], i=-L_{1}, \ldots, L_{1}\right\}$ for each $n=1,2, \ldots, N$ :

$$
E^{\prime}\left(n, k^{\prime}\right)=2 \cdot C_{L}\left[k^{\prime}\right] \cdot \operatorname{DFT}\left\{k_{n}[i], i=-L_{1}, \ldots, L_{1}\right\} .
$$

The negative frequency components are zero as a consequence of the fact that $z[n]$ is analytical; thus, the frequency resolution is doubled: instead of $L_{1}+1$ positive frequency components at $f_{k}=k / T^{\prime}, k=0,1, \ldots, L_{1}$, there are $L$ positive frequency components at the frequency points $f_{k^{\prime}}=k^{\prime} /\left(2 \cdot T^{\prime}\right), k^{\prime}=0$, $1, \ldots, L-1$, i.e., the frequency resolution of the DED is $\Delta f^{\prime}=1 /\left(2 \cdot T^{\prime}\right)$. Note that the spectrum of the original time series $x[n]$ is defined at the frequency points $f_{k}=k / T, k=0,1, \ldots, N / 2$, i.e., the frequency resolution is $\Delta f=1 / T$. Equation [26] can easily be implemented in a computer program. One can use the CZT to calculate the DFT of the odd number of data in Eq. [26]. Our CZTroutine comes from MicroWay (387FFT, version 1.00, MicroWay Inc.). Equation [26] has to be calculated for each $n$. Together the results form the DED of the equidistant analytical cardiovascular time series. The DED can be visualized graphically as a function of time and frequency by means of contour plots or three-dimensional surface plots. 


\subsection{Data Acquisition}

The applications presented in the next section were selected from several experiments in which cardiovascular parameters were studied during situations of supine rest, orthostatic stress, and controlled breathing, in healthy subjects, patients with major depressive disorder (24), and patients with autonomic dysfunctions (25). ECG, BP, and RSP were recorded continuously during supine rest (10 $\mathrm{min})$, controlled breathing by means of a metronome (16 cls $/ \mathrm{min})(3$ min), orthostatic stress ( 8 min; passive $60^{\circ}$ head-up tilt), controlled breathing (16 $\mathrm{cls} / \mathrm{min}$ ) during the $60^{\circ}$ head-up tilt $(3 \mathrm{~min})$, and a $10 \mathrm{~min}$-period of postorthostatic supine rest. The ECG was derived using a precordial lead, amplified by means of a polygraph (Nihon Kohden, Tokyo, Japan). BP was measured with a servoplethysmographic finger transducer (Finapres 2300 NIBP monitor, Ohmeda, Englewood, CO, USA). Thoracic RSP was measured with an impedance plethysmograph (Nihon Kohden, Tokyo, Japan). The signals were digitized on an IBM compatible Personal Computer (Commodore PC 60-III) equipped with an A/D converter (Advantech PC-LabCard model PCL-718). The sampling rate was $1024 \mathrm{~Hz}$ per channel. R-wave moments and R-R intervals were detected with an accuracy of $1 \mathrm{~ms}$; per R-R interval the SBP and DBP were detected with an accuracy of $0.1 \mathrm{mmHg}$; and at each R-wave moment a sample of the RSP signal was taken as explained in section 2.1, and these samples form the RSP time series. The time series were divided in periods of 3 to $5 \mathrm{~min}$ corresponding to the above mentioned experimental procedures. Of each period, the power spectrum and the DED, according to sections 2.1 and 3.1, were calculated.

\section{Applications}

\subsection{Spontaneous Cardiovascular Fluctuations during Supine Rest}

Figures 1, 2, and 3 present typical DEDs, computed from the corresponding HR, SBP, and RSP time series of a healthy subject during a 5-min period of supine rest (mean $\mathrm{HR}=65.43 \mathrm{bpm}$, variation coefficient of the interbeat intervals: $\operatorname{cov}=5.75 \%$; mean SBP: $100.1 \mathrm{mmHg}, \operatorname{cov}=4.57 \%)$. The overall spectra of HR and SBP (Figs. 1 and 2) illustrate the three relevant frequency components around $0.04 \mathrm{~Hz}$ (low), $0.1 \mathrm{~Hz}$ (mid), and around the dominant respiratory frequency at $0.3 \mathrm{~Hz}$ (high). The low frequencies $(<0.06 \mathrm{~Hz})$ of the DED of the HR and SBP time series show nonstationary fluctuations in both amplitude and frequency (Figs. 1 and 2). The mid frequency fluctuations around $0.1 \mathrm{~Hz}$ of the DED of both the HR and SBP time series are very irregular in appearance; some isolated areas of increased activity at corresponding time points are apparent, reflecting the coherence between HR and BP fluctuations in the midfrequency range. The nonstationary fluctuations of the DED of the RSP signal (Fig. 3) are reflected at the corresponding frequencies of the DEDs of the HR and SBP time series (Figs. 1 and 2, respectively) due to the respiratory modulation of vagal-mediated cardiac control (HR) and/or due to the mechanical effects of breathing (SBP). The DEDs of the HR, SBP, and RSP time series nicely reflect the different 


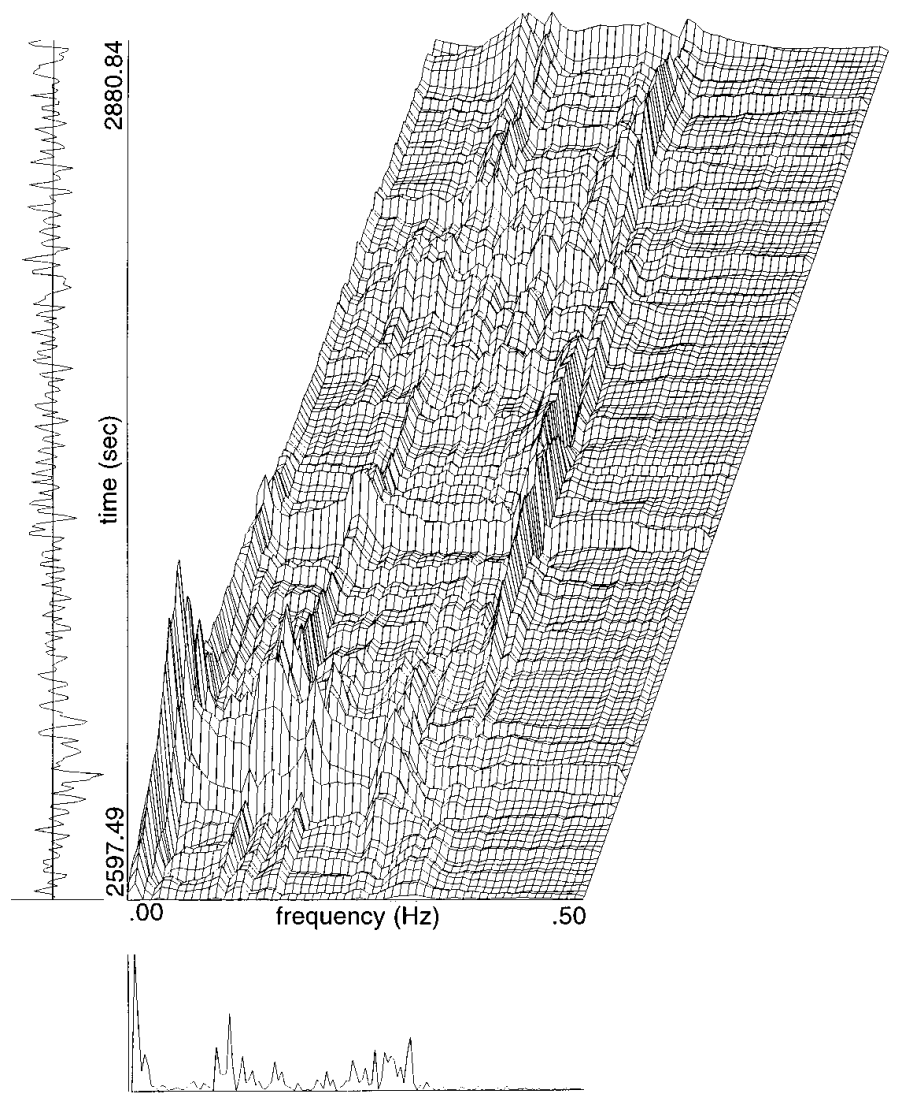

FIG. 1. DED of a nonequidistantly sampled HR time series (300 sec in length) of a healthy subject during supine rest. The abcisses is the frequency axis and the ordinate is the time axis. Along the ordinate, the raw IBI-series is shown in the time domain. Along the abcisses, the power spectral density (spectrum) of the whole time series is presented. The scale of the spectrum, in this figure and the next figures, is always equal to the maximal component in the spectrum; in this case the scale is $0.12 \mathrm{sec}^{-2} / \mathrm{Hz}$.

frequency components within short-term cardiovascular fluctuations, but they also illustrate that even within a period of several minutes and during a "stationary" situation of supine rest, complex time-related changes do occur.

\subsection{Clinical Example Illustrating Interference and Correction of Cross Terms}

The necessity to correct for cross terms in order to interpret time-frequency relationships accurately is illustrated by an example of a patient with pure autonomic failure (PAF). Patients with PAF suffer from a complete or almost complete failure of both the sympathetic and the parasympathetic nervous system, 


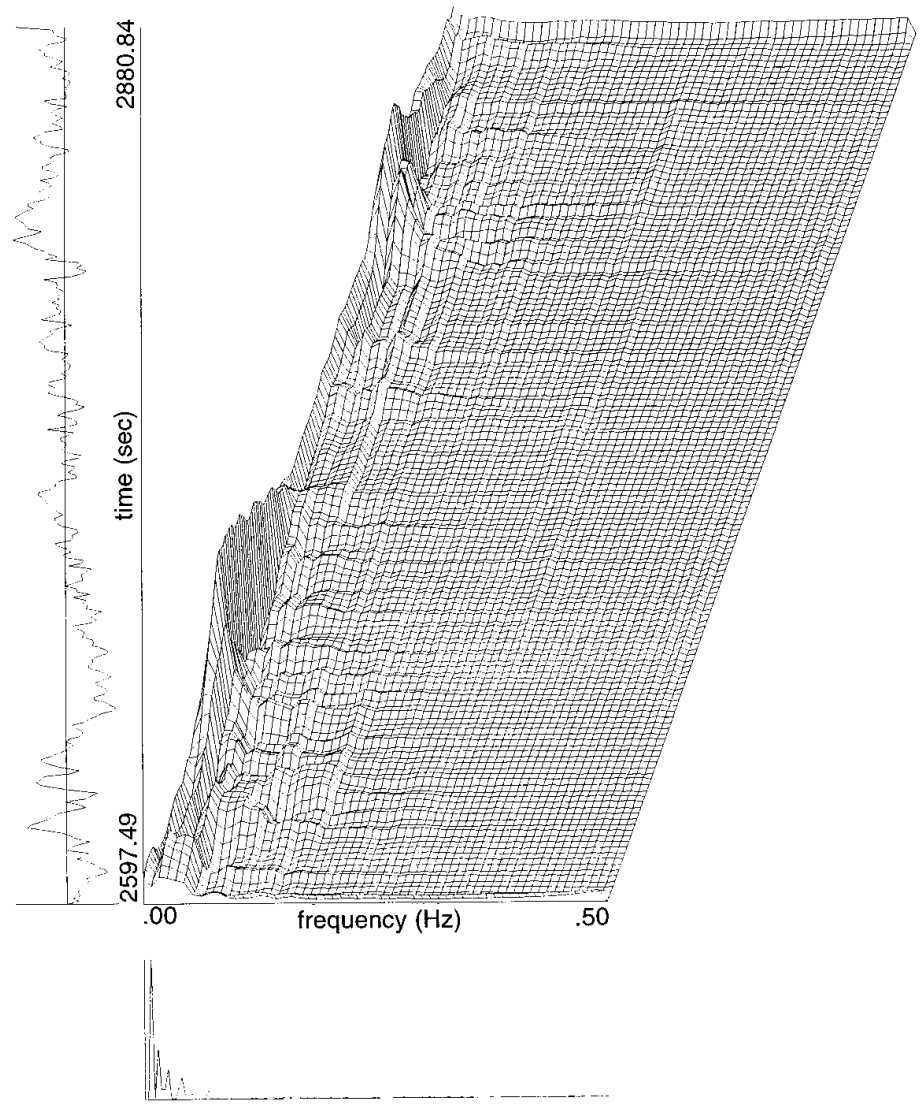

FIG. 2. DED of a nonequidistantly sampled SBP time series for the same subject and time segment as presented in Fig. 1 (see the legend of Fig. 1 for explanation of abcisses and ordinate). The scale of the spectrum is $2167.89 \mathrm{mmHg}^{2} / \mathrm{Hz}$.

resulting in dramatic reductions of both $\mathrm{HR}$ and BP variability (25). Figure 4 presents the uncorrected discrete WVD (DWVD), based on an IBI sequence of a patient with PAF during a 5-min period of supine rest (mean $\mathrm{HR}=67.3 \mathrm{bpm}$, $\operatorname{cov}=0.66 \%)$. Although the total power of the overall spectrum of the IBI sequence is low (Fig. 4), two frequency bands still are apparent: one peaks at about $0.03 \mathrm{~Hz}$, the other peaks at the respiratory frequency at about $0.2 \mathrm{~Hz}$. The fluctuations at about $0.4 \mathrm{~Hz}$ are harmonic frequencies of the respiratory frequency. Frequencies at $0.1 \mathrm{~Hz}$ are completely absent, due to the strongly diminished sympathetic tone in these patients. The DWVD does show the frequencies at $0.03,0.2$, and $0.4 \mathrm{~Hz}$, but halfway the low and respiratory peaks and halfway the respiratory peak and the peak of the harmonic frequencies, two highly irregular bands are visible: the ghost curves or cross terms (section 2.4). Notice that there is no activity at the corresponding frequencies in the overall spectrum. 


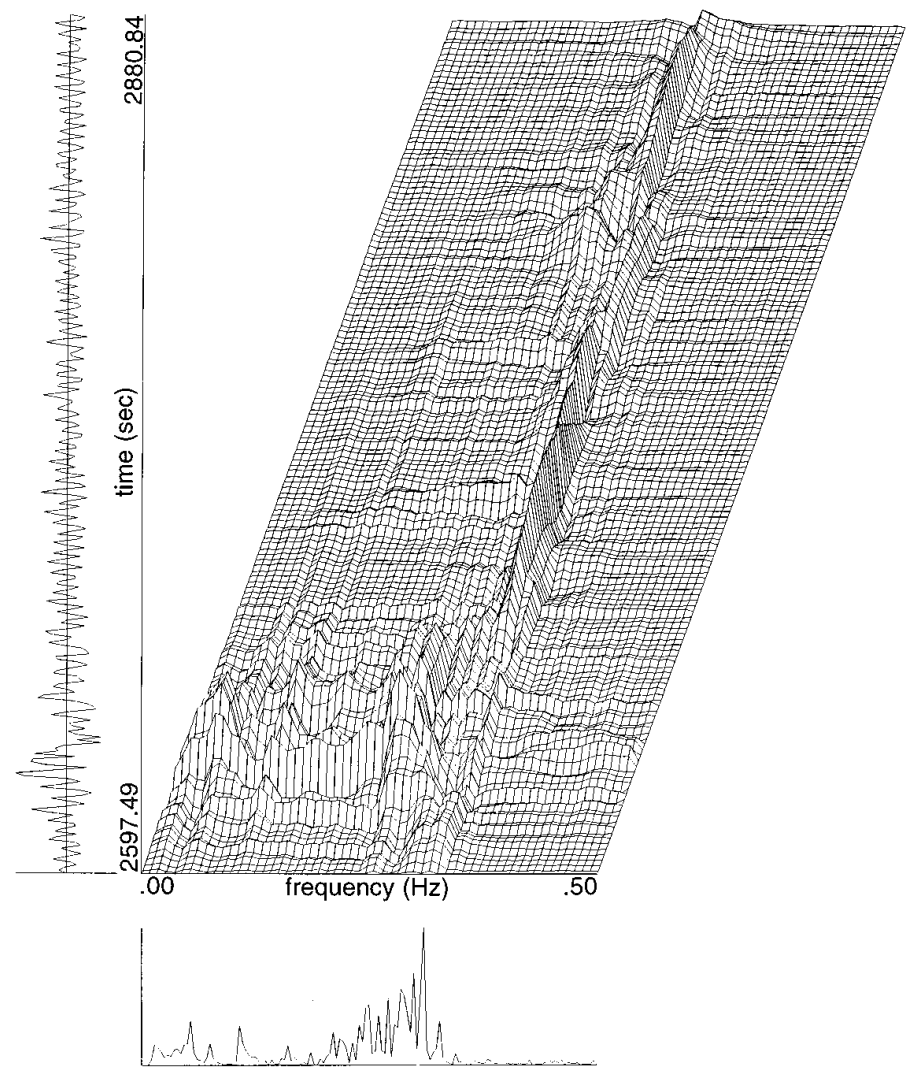

FIG. 3. DED of a nonequidistantly sampled RSP time series of the same subject and time segment as presented in Fig. 1.

Figure 5 presents the DED computed from the same IBI sequence: most of the cross terms are disappeared due to the procedures as described in section 2.4. The relevant frequency bands are now clearly represented in the timefrequency distribution. Especially in subjects with autonomic failure, where evaluation of minimal fluctuations in cardiovascular signals may have clinical relevance, adequate suppression of cross terms is an essential feature of a timefrequency distribution.

\subsection{Specific Examples of Nonstationary Time-Frequency Relationships}

1a. Respiratory modulation of HR variability. In certain situations, interpretation of overall spectra of HR (and BP) variability may lead to erroneous conclusions if there is no information available of the respiratory signal. Figures 6 and 7 illustrate the DEDs from corresponding HR and RSP time series of a depressed 


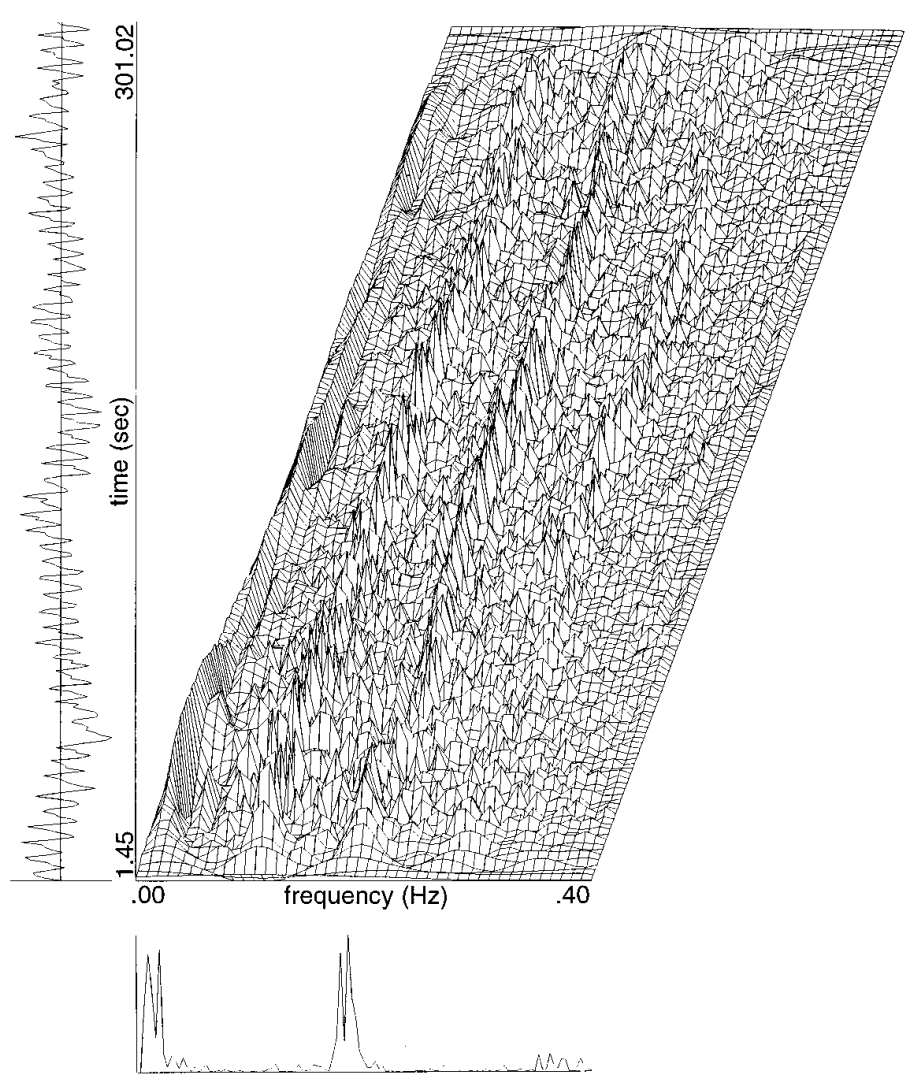

FIG. 4. DWVD of a nonequidistantly sampled IBI time series of a patient with PAF during 5 min of supine rest. The scale of the spectrum is $0.0011 \mathrm{sec}^{2} / \mathrm{Hz}$. The figure is very irregular due to the cross terms. Note the two striking cross term bands.

patient during a 4-min period of supine rest. The overall spectrum of the HR time series (Fig. 6; mean HR $=68.38 \mathrm{bpm}$, cov $=13.1 \%$ ) shows two clear broad-band frequencies: one around $0.1 \mathrm{~Hz}$, a possible reflection of sympatheticmediated processes, and one around $0.25 \mathrm{~Hz}$, reflecting respiratory-linked variations due to vagal-mediated cardiac control. The corresponding DED illustrates a sudden shift from slow to faster frequencies, suggesting a dramatic change in sympathetic modulation. However, as can be observed in Fig. 7, the corresponding respiratory signal shows a change from a slow breathing frequency (about $0.1 \mathrm{~Hz}$ ) to a faster breathing frequency $(0.18-0.22 \mathrm{~Hz})$. This time-related change is evident in the DED, whereas the overall spectrum shows the two broad-band peaks corresponding to the change in breathing frequency. Therefore, in this example, both peaks in the HR spectrum primarily reflect respiratory-linked vagal activity. 


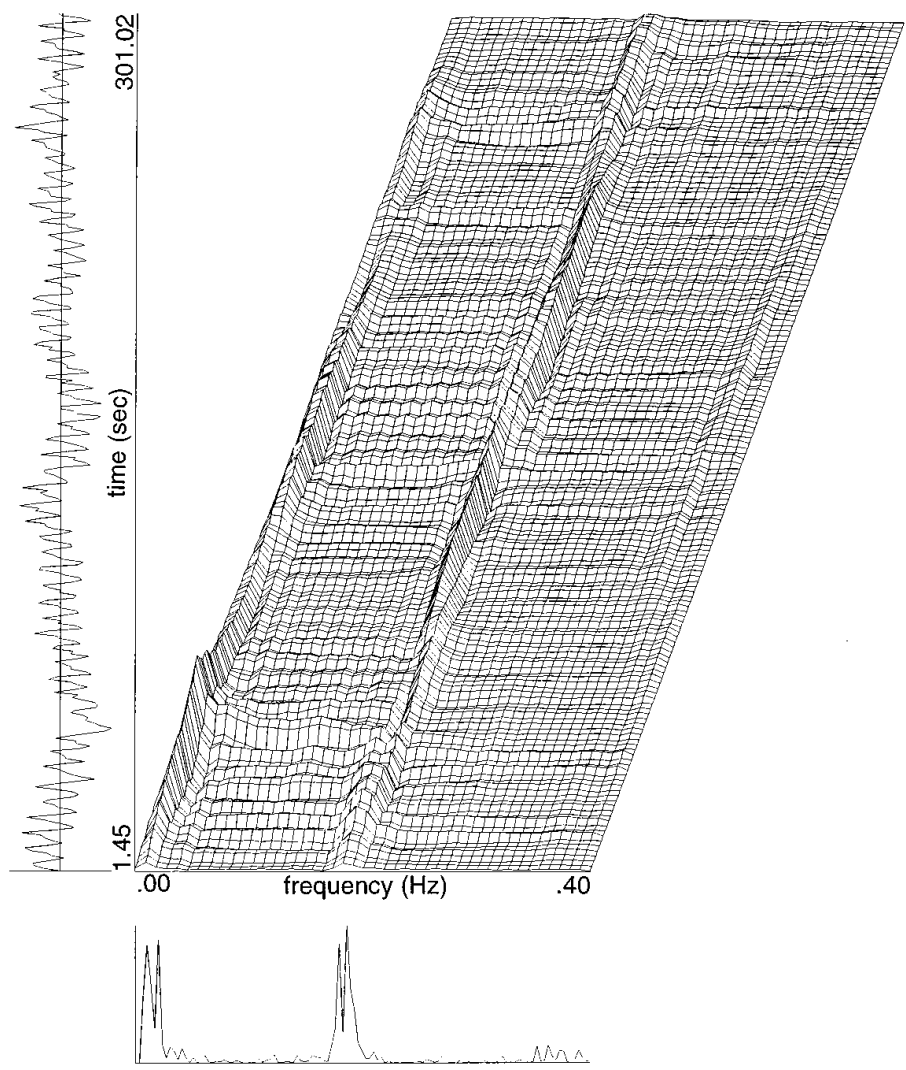

FIG. 5. DED of the same IBI time series as described in the legend of Fig. 4. The cross terms are almost disappeared due to the smoothing effect of the ED.

1b. Cardiac arrhythmia's. The DED of an IBI sequence of a depressed patient treated with the tricyclic antidepressant imipramine is presented in Fig. 8 (mean $\mathrm{HR}=79.74 \mathrm{bpm}, \mathrm{cov}=2.7 \%$ ). The IBI sequence was derived from a 4-min period of supine rest and reflects a brief episode of supraventricular cardiac arrhythmia's. This brief period of arrhythmia's affects all frequency components in the DED due to the rapid fluctuations in the successive IBI lengths. Furthermore, there appears a high peak around the Nyquist frequency in the DED. This peak is also visible in the overall spectrum of the IBI sequence and interferes greatly with the respiratory-related high frequencies. Just before and during the arrhythmic period, there is a slow trend in the IBI sequence. In the DED, this trend appears as a high peak in the low frequency range. In the spectrum, the low frequency peak is primarily caused by this temporary change. Notice the effect of the isolated arrhythmic artefacts later on in the IBI sequence. The deltalike peaks affect all frequency components in the DED. At the crosspoints 


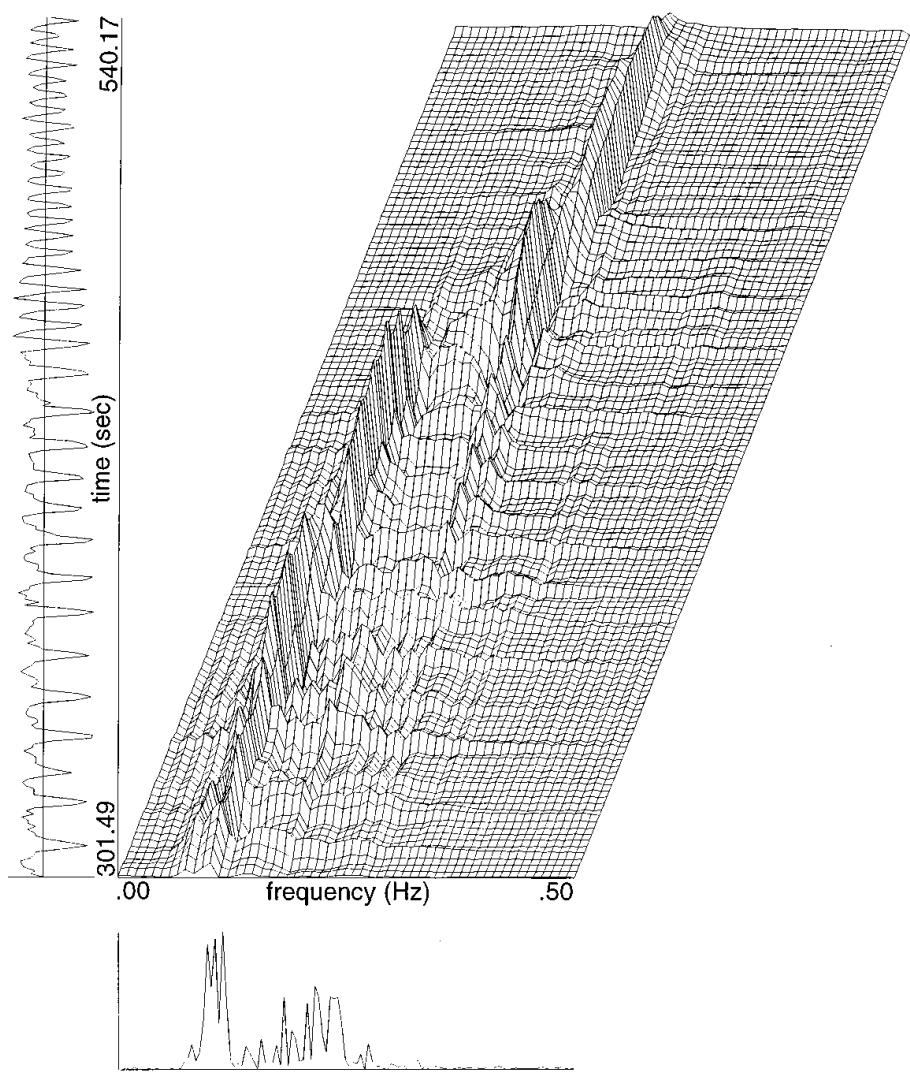

FIG. 6. DED of a nonequidistantly sampled HR time series of a depressed patient during 4 min of supine rest. The scale of the spectrum is $0.49 \mathrm{sec}^{-2} / \mathrm{Hz}$.

with the respiratory-related frequencies, the two components are superponated. Cardiac arrhythmia's have a major impact on homeostatic cardiovascular processes as reflected in HR and BP variability; this example illustrates the extent to which isolated grouped arrhythmia's disturb time-frequency distributions.

1c. Adjustments to sudden situational changes. In Fig. 9, the SBP sequence of a depressed subject reflects the initial cardiovascular reaction to the $60^{\circ}$ headup tilt procedure (mean SBP $=127.2 \mathrm{mmHg}$, cov $=4.20 \%$ ). In the DED, the respiratory-related high frequencies are apparent throughout the entire procedure. However, the transition from supine posture to passive standing causes a momentary increase in activity around $0.1 \mathrm{~Hz}$, as an indication of an increase in sympathetic tone, which is evident also in the DED. However, its reflection in the overall spectrum erroneously suggests a sustained presence of these periodicities during the entire period. The DED illustrates that the patient gets accustomed to the standing position: the activity around $0.1 \mathrm{~Hz}$ diminishes rapidly and after 


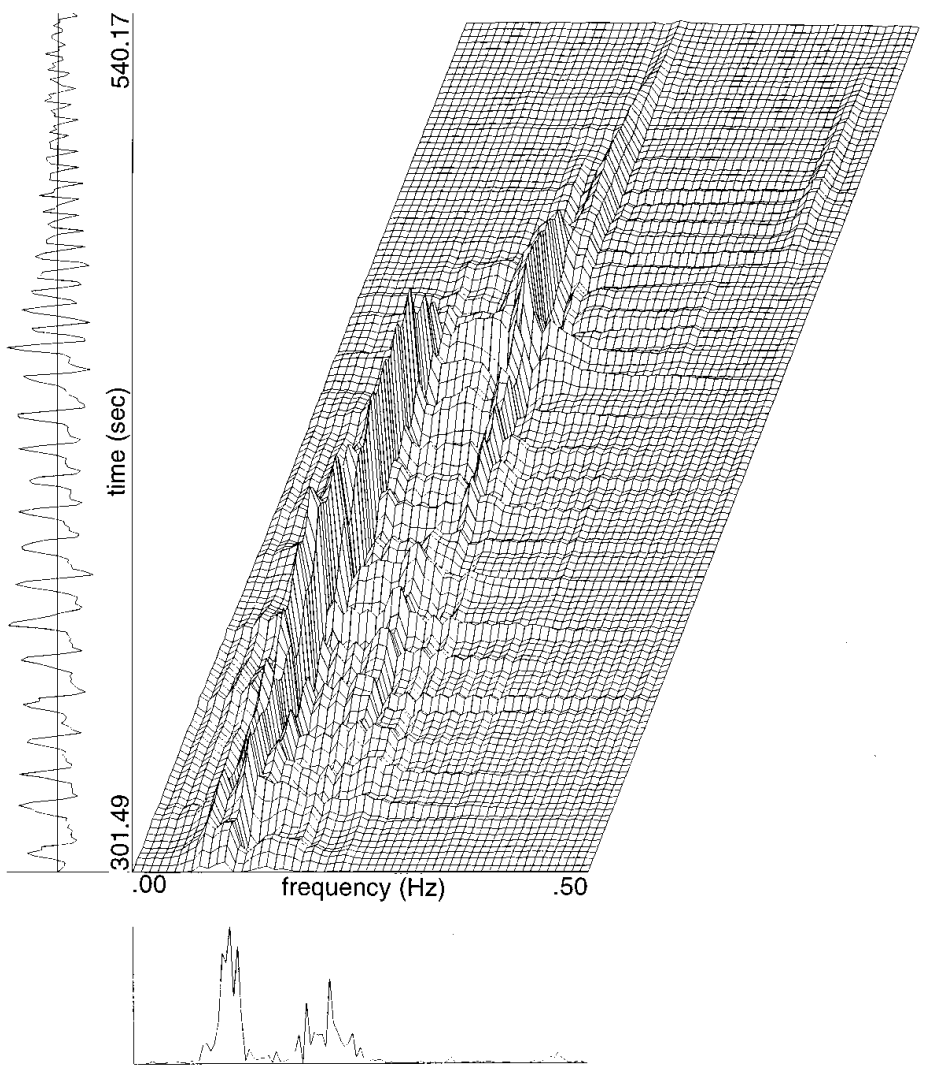

FIG. 7. DED of the RSP time series of the same subject and time segment as presented in Fig. 6. Note the sudden shift from slow to faster frequencies.

that the DED shows some isolated moments of activity at these frequencies. This example shows that initial cardiovascular adjustments to sudden situational changes can be visualized in detail by means of a DED.

\section{Discussion}

In this paper, an implementation to apply the Exponential Distribution to nonequidistantly sampled cardiovascular time series has been given. We used the methods of Rompelman (5) and Mulder (6) to transform the nonequidistantly sampled cardiovascular time series into equidistant time series. This enabled us to calculate the DED of HR as well as IBI time series. Furthermore, using the CZT enabled us to calculate the Fourier transform of time series with lengths not necessarily equal to a power of two.

The ED is a modified WVD. Implementations of the DWVD and/or DED 


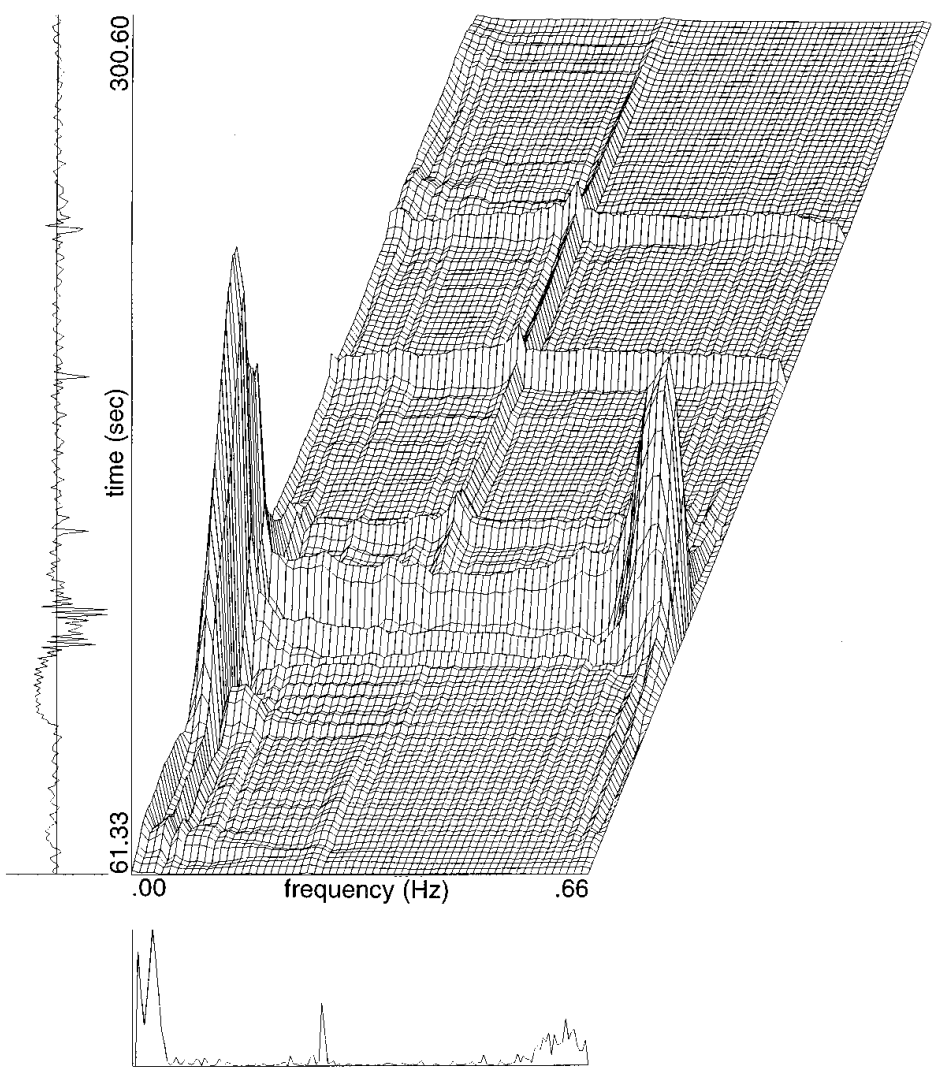

FIG. 8. DED of an IBI time series of a depressed patient during $4 \mathrm{~min}$ of supine rest. The scale of the spectrum is $0.0095 \mathrm{sec}^{2} / \mathrm{Hz}$.

have been discussed previously by Martin and Flandrin (26), Chester (27), Boashash and Black (18), Sun et al. (28), Boashash and Reilly (23). A method to suppress cross terms in a DVD has been discussed by Martin and Flandrin (26). They used a symmetric normalized time window and a time smoothing function. The effect of the time window is smoothing of the WVD in the frequency direction; thus, it will also spread out the autoterms. The effect of the smoothing function is a reduction of the cross terms, but it has also a smoothing effect on the autoterms in the time direction. Therefore, the implementation of the DWVD by Martin and Flandrin does not satisfy the properties P3-P8 (section 2.3). Novak and Novak (15) applied the DWVD, using FFT techniques, to IBI, SBP, DBP, and RSP time series, each time series linearly interpolated to an equidistant time series. They implemented the method of Martin and Flandrin to correct for cross terms, using a computation algorithm of Peyrin and Prost (29). We employed the ED because it has certain advantages over the aforementioned method: the 


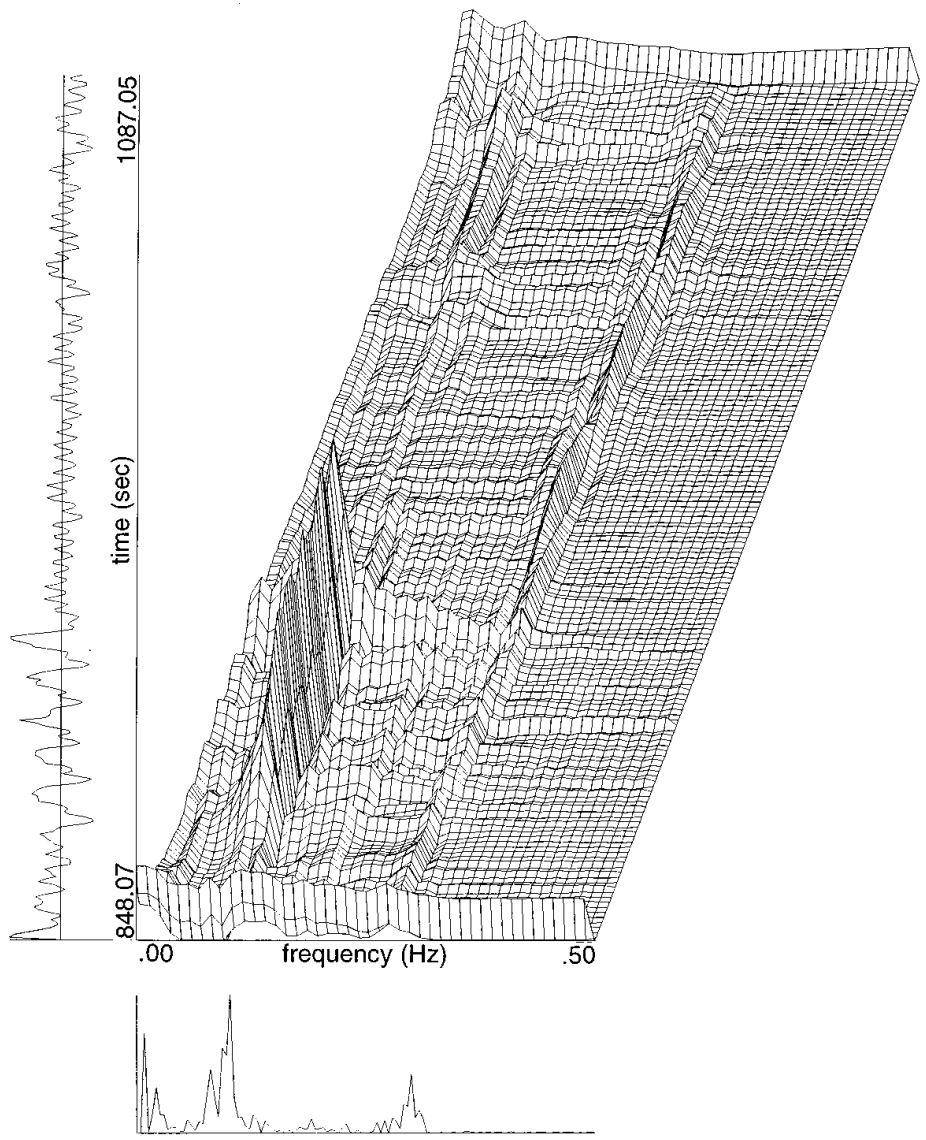

FIG. 9. DED of a SBP time series of a depressed patient during 4 min of head up tilt. The scale of the spectrum is $767.11 \mathrm{mmHg}^{2} / \mathrm{Hz}$. The initial cardiovascular reaction to the head up tilt is most prominent in the frequency components around $0.1 \mathrm{~Hz}$.

ED satisfies the advocated properties P1-P6 (section 2.3) for TFDs, for all values of $\sigma$. When the smoothing effect is too large, i.e., small $\sigma(\sigma \leq 1 ; 16)$, the autoterms and the components in the time direction will be spread noticeably. Thus, P7 and P8 will not be satisfied. If $\sigma$ is sufficiently large $(\sigma>1)$ then the ED will approximately satisfy properties P7 and P8 (14). Furthermore, for increasing $\sigma$, the ED will become approximately equal to the WVD. According to Choi and Williams, a good choice of $\sigma$ will be found between 0.1 and 10 . Our DED examples of cardiovascular time series, with $\sigma=2$, show that the cross term suppression by the ED is satisfactory without disturbing the autoterms too much. Our applications illustrated the potential of the ED to study timefrequency relationships of and between cardiovascular time series. Complex 
time-varying aspects of the relevant cardiovascular frequency components of HR, IBI, BP, and RSP time series could be made visible by means of the ED. Therefore, the ED can be an important aid to describe and interpret the complex dynamics of autonomic regulatory processes.

\section{REFERENCES}

1. SAyers, B. McA. Analysis of heart rate variability. Ergonomics 16, 17-32 (1973).

2. Akselrod, S., Gordon, D., Madwed, J. B., Snidman, N. C., Shannon, D. C., and Cohen, R. J. Hemodynamic regulation: Investigation by spectral analysis. Am. J. Physiol. 249, M867M875 (1985).

3. Tulen, J. H. M., Smeets, F. M. L., Man In 'T Veld, A. J., Van Steenis, H. G., Van De Wetering, B. J. M., And Moleman, P. Cardiovascular variability after clonidine challenge: Assessment of dose-dependent temporal effects by means of spectral analysis. J. Cardiovasc. Pharmacol. 22, 112-119 (1993).

4. Tulen, J. H. M., Man In 'T Veld, A. J., Van Roon, A. M., Moleman, P., Van Steenis, H. G., Blankestijn, P. J., And Boomsma, F. Spectral analysis of hemodynamics during infusions of epinephrine and norepinephrine in men. J. Appl. Physiol. 76(5), 1914-1921 (1994).

5. Rompelman, O. Spectral analysis of heart-rate variability. In "Psychophysiology of Cardiovascular Control” J. F. Orlebeke, G. Mulder, and L. J. P. Van Doornen, Eds.), pp. 315-331. Plenum Press, New York/London, 1985.

6. Mulder, L. J. M. Assessment of cardiovascular reactivity by means of spectral analysis. Ph.D. Thesis, University of Groningen, Groningen, 1988.

7. Van Steenis, H. G., Tulen, J. H. M., And Mulder, L. J. M. Heart rate variability spectra based on non-equidistant sampling: The spectrum of counts and the instantaneous heart rate spectrum. Med. Eng. Phys. 16, 355-362 (1994).

8. Parati, G., Castiglioni, P., Di Renzo, M., Omboni, S., Pedotti, A., and Mancia, G. Sequential spectral analysis of 24-hour blood pressure and pulse interval in humans. Hypertension 16, 414-421 (1990).

9. Wigner, E. On the quantum correction for thermodynamic equilibrium. Phys. Rev. 40, 749759 (1932).

10. VIlle, J. Théorie et applications de la notion de signal analytique. Cables et Transmission, $2^{e} A$ 1, 61-74 (1948).

11. Clatassen, T. A. C. M., and Mecklenbräuker, W. F. G. The Wigner distribution-A tool for time-frequency signal analysis. Part I: Continuous-time signals. Philips Res. J. 35, 217-250 (1980).

12. Clanassen, T. A. C. M., and Mecklenbräuker, W. F. G. The Wigner distribution-A tool for time-frequency signal analysis. Part II: Discrete-time signals. Philips Res. J. 35, 276-300 (1980).

13. Boashash, B. Time-frequency signal analysis. In "Advances in Spectrum Analysis and Array Processing” (S. Haykin, Ed.), Vol. I, pp. 418-517. Prentice Hall, Englewood Cliffs, NJ, 1991.

14. Cohen, L. Time-frequency analysis. Prentice Hall, Englewood Cliffs, NJ, 1995.

15. Novak, P., And NovaK, V. Time/frequency mapping of the heart rate, blood pressure and respiratory signals. Med. Biol. Eng. Comput. 31, 103-110 (1993).

16. Choi, H. I., AND Williams, W. J. Improved time-frequency representation of multicomponent signals using exponential kernels. IEEE Trans. Acoust., Speech, Signal Process. ASSP-37, 862871 (1989).

17. Bendat, J. S., And Piersol, A. G. "Random Data, Analysis and Measurement Procedures," 2nd ed. Wiley, New York, 1986.

18. Boashash, B., and Black, P. J. An efficient real-time implementation of the Wigner-Ville distribution. IEEE Trans. Acoust., Speech, Signal Process. ASSP-33, 1611-1618 (1987).

19. Boashash, B. Note on the use of the Wigner distribution for time-frequency signal analysis. IEEE Trans. Acoust., Speech, Signal Process. ASSP-36, 1518-1521 (1988).

20. Kоotsookos, P. J., Lovell, B. C., And Boashash, B. A unified approach to the STFT, TFD's and instantaneous frequency. IEEE Trans. Signal Process. 40, 1971-1982 (1992). 
21. Allen, J. B. And Rabiner, L. R. A unified approach to short-time Fourier analysis and synthesis. Proc. IEEE 65, 1558-1564 (1977).

22. Oppenheim, A. V., And Schafer, R. W. "Digital Signal Processing. Prentice-Hall, Englewood Cliffs, NJ, 1975.

23. Boashash, B., And Reilly, A. Algorithms for time-frequency signal analysis. In "Time-Frequency Signal Analysis" (B. Boashash, Ed.), pp. 163-181. Longman Cheshire, South Melbourne, Australia, 1992.

24. Tulen, J. H. M., Bruijn, J. A., De Man, K. J., van Den Meiracker, A. H., Pepplinkhuizen, L., AND MAN IN 'T Veld, A. J. Cardiovascular variability in major depressive disorder and effects of imipramine or mirtazapine (ORG 3770). J. Clin. Psychopharmacol., in press.

25. Van Den Meiracker, A. H., Tulen, J. H. M., Man In 'T Veld, A. J., Balk, A. H. M. M., Van Steenis, H. G., And Schalekamp, M. A. D. H. Blood pressure and heart rate variability in clinical models of cardiac denervation, sympathicolysis and sympathico- and parasympathicolysis. J. Hypertens. 11 (suppl 5), S152-S153 (1993).

26. Martin, M., And Flandrin, P. Wigner-Ville spectral analysis of nonstationary processes. IEEE Trans. Acoust., Speech, Signal Process. ASSP-33, 1461-1470 (1985).

27. Chester, D. Discrete Wigner implementations. 1986 Int. Symp. Circuit Syst. Proc., San Jose, 38-41 (1986).

28. Sun, M., Li, C. C., Sekhar, L. N., and Sclabassi, R. J. Efficient computation of the discrete pseudo-Wigner distribution. IEEE Trans. Acoust., Speech, Signal Process. ASSP-37, 17351742 (1989).

29. Peyrin, F., And Prost, R. A unified definition for the discrete-time, discrete-frequency and discrete-time/frequency Wigner distribution. IEEE Trans. Acoust., Speech, Signal Process. ASSP34, 858-867 (1986). 